\title{
A THEORETICAL INVESTIGATION OF AN ASYMMETRIC PLANING HULL AT INFINITE FROUDE NUMBER
}

\author{
E. M. CASLING
}

(Received 20 March 1979)

(Revised 8 January 1980)

\begin{abstract}
The extent to which an asymmetric low-aspect-ratio flat ship is wetted when planing at infinite Froude number is investigated, with emphasis placed on its relationship with the shape of the hull. Two cases are considered. First the hull is assumed to have two laterally-asymmetric leading edges and, secondly, the hull is assumed to be yawed sufficiently for one of the leading edges to become a trailing edge. In the first case, the relationship involves a pair of coupled integral equations, but in the second case there is a complication by the occurrence of hull-wake interaction.
\end{abstract}

\section{Introduction}

As distinct from the displacement hulls of ordinary ships, which are supported by buoyancy, most of the weight of a planing boat is supported by the hydrodynamic lift force resulting from the upward reaction of the fluid on the moving body. Planing usually occurs when the boat concerned is a high-speed craft of comparatively small weight. The flow is essentially a potential flow and the pressure distribution on the bottom of the hull may be determined without considering viscous forces. Planing is a field which has attracted considerable attention since the first experiment with a flat planing surface was carried out in 1912 (see Baker and Millar [1]), and both theoretical and experimental investigations have been undertaken by a large number of authors. A bibliography and brief review of some of the more important papers is given in Casling [3].

In most discussions of planing problems, it is usually assumed that the hull is laterally-symmetric. However, in practice, this is not always the case. For example, when a catamaran is planing with one hull out of the water, the wetted region of the other hull can no longer be considered to be symmetric with respect to the direction in which it is moving. Similarly, when a boat moving at high speed is turning, until 
the turn is completed, the waterplane is yawed with respect to the direction of its motion. A surfboard is another example of a laterally-asymmetric planing surface (see Hornung and Killen [4]). It is interesting to calculate the forces and moments acting on such hulls in order to determine, in the case of the catamaran, for example, whether the hull tends to "right" itself (that is, become laterally-symmetric) or whether it shows a tendency to turn right over.

Two classes of problems are considered in this paper. Firstly, the case when the boat has two leading edges which are asymmetric with respect to the direction of motion is discussed. The second case concerns hulls which are sufficiently yawed for one of the leading edges to become a trailing edge, thus generating a wake forward of the stern of the hull. The presence of the wake leads to an integral equation for the velocity potential, which is unknown in that region, and adds considerably to the mathematical difficulty of the problem. However, Tuck [6] obtained an almost identical equation for yawed slender wings and presented a method for its solution, with an analytic result possible in particular cases.

For both problems, an expression for the free-surface elevation is derived, which leads to a pair of coupled integral equations in the first case and a single integral equation in the second, relating section shape, waterplane shape and longitudinal hull slope. These results are contained in Casling [3].

\section{Mathematical formulation}

A low-aspect-ratio flat ship is assumed to be moving with speed $U$ in the negative $s$-direction, the origin of the coordinate system $(x, y, s)$ being fixed to the bow (see Fig. 1). The fluid is assumed to be ideal, so that viscosity and surface tension are neglected, and the undisturbed free surface coincides with the plane $y=0$. Thus the flow may be assumed to be irrotational, with the velocity field given by

$$
\mathbf{q}=\nabla \Phi=\nabla(U s+\phi),
$$

where $\phi$ is the perturbation velocity potential.

The surface of the hull is given by

$$
y=\eta(x, s)
$$

for $-a(s)<x<b(s)$, since the waterplane is asymmetric, and for $s<L$, where $L$ is the wetted length of the hull. Note that $a(s)$ and $b(s)$ are both assumed to be nonnegative (that is, $a(s) \geqslant 0, b(s) \geqslant 0$ for $0 \leqslant s \leqslant L)$ and strictly monotone-increasing functions of $s$, and that the leading edges of the hull are given by $x=-a(s)$ and $x=b(s)$ (see Fig. 2). It is also assumed that $\eta(x, s)$ is a strictly monotone-decreasing 


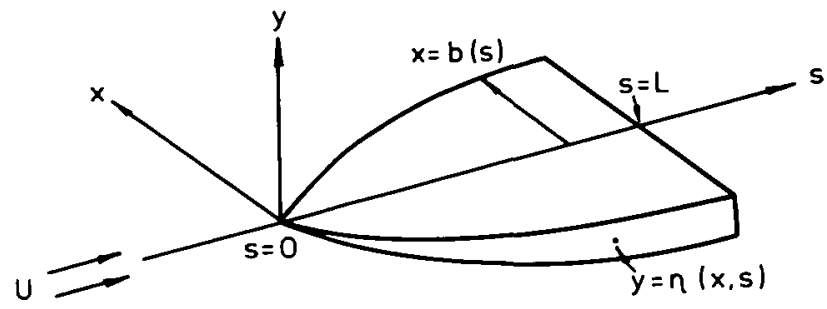

Fig. 1. Coordinate system.

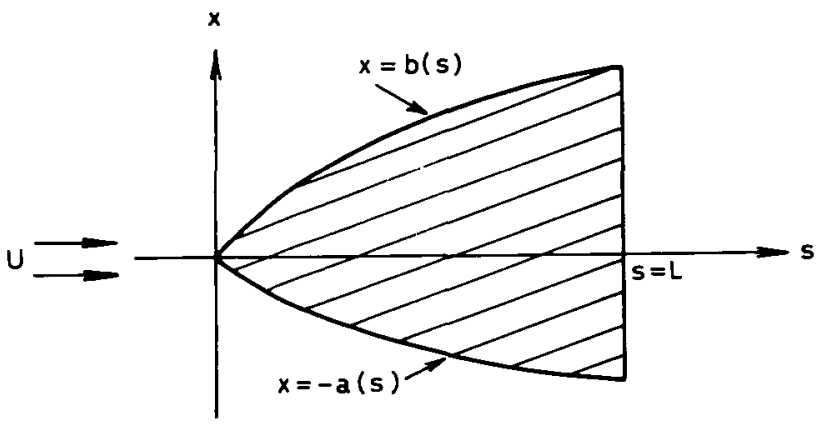

Fig. 2. Asymmetric waterplane.

function of $s$. Outside the hull surface, equation (2.1) describes the free-surface elevation caused by the ship's motion. Therefore $\phi$ satisfies the full threedimensional Laplace equation

$$
\phi_{x x}+\phi_{y y}+\phi_{s s}=0
$$

in the region $y<\eta(x, s)$.

The exact hull boundary condition, of no flow normal to the body, is

$$
\phi_{y}=\left(U+\phi_{s}\right) \eta_{s}+\phi_{x} \eta_{x} \quad \text { on } y=\eta(x, s) .
$$

Outside the hull surface, equation (2.2) is a kinematic condition on the unknown free surface.

The dynamic free surface condition, for zero gravity (that is, for infinite Froude number), is

$$
\frac{P}{\rho}+U \phi_{s}+\frac{1}{2}|\nabla \phi|^{2}=0 \quad \text { on } y=\eta(x, s),
$$

where $P$ is defined to be the excess of pressure over atmospheric at the free surface. Two more conditions are necessary. A Kutta-like condition, which ensures that the free surface leaves any sharp trailing edge smoothly, is needed. For example, the pressure should reduce to atmospheric at any such edge. In addition, a radiation 
condition at infinity, namely $\phi_{x}, \phi_{y} \rightarrow 0$ as $x^{2}+y^{2}+s^{2} \rightarrow \infty$ in the lower half plane, is required.

The ship is assumed to be flat as well as slender, with

$$
D \ll B \ll L,
$$

where $D$ is the draft and $B$ the beam of the ship. That is, if

$$
D=O(\alpha) . L \text { and } B=O(\varepsilon) . L,
$$

for small parameters $\alpha$ and $\varepsilon$, then

$$
\alpha \ll \varepsilon .
$$

When first the small-draft and then the low-aspect-ratio approximation is made, using standard perturbation techniques, equations (2.2) and (2.3) reduce respectively to

$$
\phi_{y}=\mathrm{U} \eta_{s} \quad \text { on } y=0
$$

and

$$
\frac{P}{\rho}+U \phi_{s}=0 \quad \text { on } y=0 .
$$

These linearized conditions are applied on $y=0$ because, as $\alpha \rightarrow 0$, the hull reduces to its projection onto the plane $y=0$ and, since the ship is slender, it is not possible, in general, to satisfy the Kutta condition at the trailing edge. The function $\phi$ is now the potential for the cross-flow problem in the $(x, y)$-plane and satisfies the twodimensional Laplace equation

$$
\phi_{x x}+\phi_{y y}=0
$$

in the region $y<0$. The problem for $\phi$ is shown in Fig. 3 .

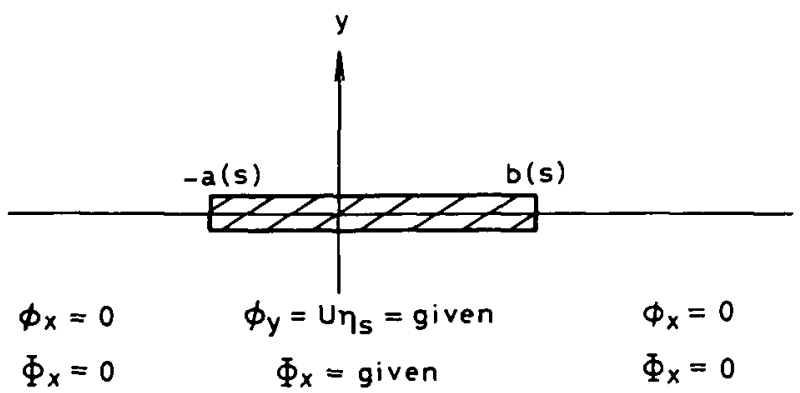

Fig. 3. Cross-flow plane.

By considering the linearized problem, some of the details of planing flow, for example, the spray sheet, are neglected. However, Wagner [7] has shown that this phenomenon may be represented by a square-root singularity in the pressure at the leading edge, a result which is incorporated into the solution of the problem. 


\section{A solution of the problem}

There are many ways in which the problem just defined may be solved. One of the easiest methods involves the use of complex variable techniques.

The complex function $\Omega(z)$ is defined by

$$
\begin{aligned}
\Omega(z) & =\Phi_{x}(x, y, s)-i \Phi_{y}(x, y, s) \\
& =(z+a(s))^{\frac{1}{2}}(z-b(s))^{\frac{1}{2}} w(z),
\end{aligned}
$$

where $z=x+i y$ and $w(z)=\phi_{x}(x, y, s)-i \phi_{y}(x, y, s)$ is the complex velocity at station $s$, since there is an inverse square-root singularity in the fluid velocity at the leading edges, $x=-a(s)$ and $x=b(s)$. The branches of the square-root functions are taken so that

and

$$
(z-b(s))^{\frac{1}{2}} \rightarrow-i(b(s)-x)^{\frac{1}{2}} \text { as } y \rightarrow 0-, \text { for } x<b(s),
$$

$$
(z+a(s))^{\frac{1}{t}} \rightarrow-i(-a(s)-x)^{\frac{1}{2}} \text { as } y \rightarrow 0-, \text { for } x<-a(s) .
$$

Thus, in the limit as $y \rightarrow 0-$, equation (3.1) may be written

$$
\Phi_{x}-i \Phi_{y}=\left\{\begin{array}{lc}
(x-b(s))^{\frac{1}{2}}(x+a(s))^{\frac{1}{2}}\left(\phi_{x}-i \phi_{y}\right), & x>b(s), \\
-i(b(s)-x)^{\frac{1}{2}}(x+a(s))^{\frac{1}{2}}\left(\phi_{x}-i \phi_{y}\right), & -a(s)<x<b(s), \\
-(b(s)-x)^{\frac{1}{2}}(-a(s)-x)^{\frac{1}{2}}\left(\phi_{x}-i \phi_{y}\right), & x<-a(s) .
\end{array}\right.
$$

The problem for $\Phi$ is shown on Fig. 3.

Since $\Omega(z)$ is an analytic function in the lower half-plane and tends to zero as $|z| \rightarrow \infty$, from the radiation condition on $\phi_{x}$ and $\phi_{y}$, Cauchy's theorem implies that

$$
\Phi_{x}(x, 0-, s)-i \Phi_{y}(x, 0-, s)=\frac{1}{\pi i} \int_{-\infty}^{\infty} \frac{d \xi}{x-\xi}\left(\Phi_{x}(\xi, 0-, s)-i \Phi_{y}(\xi, 0-, s)\right),
$$

where the integral is interpreted as a Cauchy principal-value integral. In particular, since $\Phi_{x}$ is known everywhere,

$$
\Phi_{y}(x, s)=\frac{1}{\pi} \int_{-a(s)}^{b(s)} \frac{d \xi}{x-\xi} \Phi_{x}(\xi, s)
$$

may be determined everywhere, there being no contribution to the integral for $\xi>b(s)$ or $\xi<-a(s)$ (see Fig. 3). Expressing equation (3.2) in terms of the original functions $\phi_{x}$ and $\phi_{y}$,

$$
\left.\begin{array}{l}
\phi_{y}(x, s) \\
\phi_{x}(x, s) \\
\phi_{y}(x, s)
\end{array}\right\}=\frac{1}{\pi}\left\{\begin{array}{ll}
\frac{-1}{(x+a(s))^{\frac{1}{2}}(x-b(s))^{\frac{1}{2}}} \\
\frac{-1}{(x+a(s))^{\frac{1}{2}}(b(s)-x)^{\frac{1}{2}}} \\
\frac{1}{(-a(s)-x)^{\frac{1}{2}}(b(s)-x)^{\frac{1}{2}}}
\end{array}\right\} I_{1}(x, s), \quad-a(s)<x<b(s),
$$


where

$$
I_{1}(x, s)=\int_{-a(s)}^{b(s)} \frac{d \xi}{x-\xi} \phi_{y}(\xi, s)(b(s)-\xi)^{\frac{1}{2}}(\xi+a(s))^{\frac{1}{2}} .
$$

Since $\phi_{y}(x, s)$ is now a known function everywhere, the slope of the free surface, $\eta_{s}(x, s)$ is also known, from equation (2.4), and the free-surface elevation, $\eta(x, s)$, may be described by the following expressions :

$$
\eta(x, s)= \begin{cases}-\frac{1}{\pi} \int_{0}^{s} \frac{d \sigma}{(x+a(\sigma))^{\frac{1}{2}}(x-b(\sigma))^{\frac{1}{2}}} I_{2}(x, s), & x>b(s) \\ \int_{0}^{s} d \sigma \eta_{\sigma}(x, \sigma)+c_{1}(x), & 0<x<b(s) \\ \int_{0}^{s} d \sigma \eta_{\sigma}(x, \sigma)+c_{2}(x), & -a(s)<x<0 \\ \frac{1}{\pi} \int_{0}^{s} \frac{d \sigma}{(-a(\sigma)-x)^{\frac{1}{2}}(b(\sigma)-x)^{\frac{1}{2}}} I_{2}(x, s), & x<-a(s),\end{cases}
$$

where

$$
\begin{aligned}
c_{1}(x)=- & \frac{1}{\pi} \int_{0}^{s_{0}(x)} \frac{d \sigma}{(x+a(\sigma))^{\frac{1}{2}}(x-b(\sigma))^{\frac{1}{2}}} \int_{-a(\sigma)}^{b(\sigma)} \frac{d \xi}{x-\xi} \\
& \times\left(\eta_{\sigma}(\xi, \sigma)-\eta_{\sigma}(x, \sigma)\right)(\xi+a(\sigma))^{\frac{1}{2}}(b(\sigma)-\xi)^{\frac{1}{2}} \\
& -x \int_{0}^{s_{0}(x)} d \sigma \frac{\eta_{\sigma}(x, \sigma)}{(x+a(\sigma))^{\frac{1}{2}}(x-b(\sigma))^{\frac{1}{2}}} \\
& +\frac{1}{2} \int_{0}^{s_{0}(x)} d \sigma \frac{\eta_{\sigma}(x, \sigma)(b(\sigma)-a(\sigma))}{(x+a(\sigma))^{\frac{1}{2}}(x-b(\sigma))^{\frac{1}{2}}} \\
c_{2}(x)=\frac{1}{\pi} \int_{0}^{s_{1}(x)} & \frac{d \sigma}{(-a(\sigma)-x)^{\frac{1}{2}}(b(\sigma)-x)^{\frac{1}{2}}} \\
& \times \int_{-a(\sigma)}^{b(\sigma)} \frac{d \xi}{x-\xi}\left(\eta_{\sigma}(\xi, \sigma)-\eta_{\sigma}(x, \sigma)\right)(\xi+a(\sigma))^{\frac{1}{2}}(b(\sigma)-\xi)^{\frac{1}{2}} \\
& +x \int_{0}^{s_{1}(x)} d \sigma \frac{\eta_{\sigma}(x, \sigma)}{(-a(\sigma)-x)^{\frac{1}{2}}(b(\sigma)-x)^{\frac{1}{2}}} \\
& -\frac{1}{2} \int_{0}^{s_{1}(x)} d \sigma \frac{\eta_{\sigma}(x, \sigma)(b(\sigma)-a(\sigma))}{(-a(\sigma)-x)^{\frac{1}{2}}(b(\sigma)-x)^{\frac{1}{2}}} \\
I_{2}(x, s) & =\int_{-a(s)}^{b(s)} \frac{d \xi}{x-\xi} \eta_{s}(\xi, s)(\xi+a(s))^{\frac{1}{2}}(b(s)-\xi)^{\frac{1}{2}},
\end{aligned}
$$


$s_{0}(x)$ is the station at which $x=b(s)$, and $s_{1}(x)$ is the station at which $x=-a(s)$.

A relationship between the physical characteristics of the hull and the shape of its wetted area when it is moving has emerged, in which only two variables can be fixed, the third being determined by the relationship. This result is not unexpected, because a similar property has already been observed for symmetric planing hulls (see Casling [2]). In this case, it takes the form of a pair of coupled integral equations, given in equations (3.5) and (3.6). If the water-plane shape, described by $x=h(s)$ and $x=-a(s)$, and the longitudinal hull slope, $\eta_{s}(x, s)$, are known, then the complete hull shape, determined by $y=c_{1}(x)$ for $x>0$ and $y=c_{2}(x)$ for $x<0$, is fixed by these equations. If $\eta(x, s)$ is assumed to be completely defined, as in equation (3.4) for $-a(s)<x<b(s)$, then the pair of integral equations must be inverted simultaneously to find the unknown waterplane shape, that is, to find the functions $x=b(s)$ and $x=-a(s)$. There seems to be little hope for an analytic result in this case.

However, the indirect problem (that is, the one in which the waterplane is fixed) may be used in an inverse manner to produce required hull shapes. For example, suppose $\eta_{s}(x, s)$ is independent of $x$ and $s$, that is,

$$
\eta_{s}(x, s)=-\gamma,
$$

for some small constant $\gamma$. Then equations (3.5) and (3.6) become

$$
c_{1}(x)=\gamma x \int_{0}^{s_{0}(x)} \frac{d \sigma}{(x+a(\sigma))^{\frac{1}{2}}(x-b(\sigma))^{\frac{1}{2}}}-\frac{1}{2} \gamma \int_{0}^{s_{0}(x)} d \sigma \frac{(b(\sigma)-a(\sigma))}{(x+a(\sigma))^{\frac{1}{2}}(x-b(\sigma))^{\frac{1}{2}}}
$$

and

$$
c_{2}(x)=-\gamma x \int_{0}^{s_{1}(x)} \frac{d \sigma}{(-a(\sigma)-x)^{\frac{1}{2}}(b(\sigma)-x)^{\frac{1}{2}}}+\frac{1}{2} \gamma \int_{0}^{s_{1}(x)} d \sigma \frac{(b(\sigma)-a(\sigma))}{(-a(\sigma)-x)^{\frac{1}{2}}(b(\sigma)-x)^{\frac{1}{2}}}
$$

respectively. If it is further assumed that the waterplane is triangular, with

$$
b(s)=\beta s \quad \text { and } \quad a(s)=\alpha s
$$

for small constants $\alpha$ and $\beta$, then

$$
c_{1}(x)=c_{1} x \text { and } c_{2}(x)=c_{2} x
$$

where

$$
c_{1}=\gamma\left[\left(\frac{\pi}{2}+\sin ^{-1}\left(\frac{\alpha-\beta}{\alpha+\beta}\right)\right)(\beta+\alpha)^{2} / 4-(\beta-\alpha)(\alpha \beta)^{ \pm} / 2\right] /(\alpha \beta)^{3 / 2}
$$


and

$$
c_{2}=-\gamma\left[\left(\frac{\pi}{2}-\sin ^{-1}\left(\frac{\alpha-\beta}{\alpha+\beta}\right)\right)(\beta+\alpha)^{2} / 4+(\beta-\alpha)(\alpha \beta)^{\frac{1}{2}} / 2\right] /(\alpha \beta)^{3 / 2}
$$

are constants. Thus the section shape is also triangular. So the extent to which a given hull with triangular sections is wetted may be determined by altering the values of $\alpha$ and $\beta$ until the desired values of $c_{1}$ and $c_{2}$ are obtained. A similar process may be used for other hull shapes.

\section{Forces and moments}

From equation (3.3), $\phi_{x}(x, s)$ may be determined for $-a(s)<x<b(s)$. Therefore expressions may be derived for the forces and moments acting on the hull and the position of the centre of pressure may be calculated.

The lift force, $F_{Y}$, on the hull is given by

$$
\begin{aligned}
F_{Y} & =\int_{0}^{L} d s \int_{-a(s)}^{b(s)} d x P(x, s) \\
& =\int_{-a(L)}^{0} d x \int_{s_{1}(x)}^{L} d s P(x, s)+\int_{0}^{b(L)} d x \int_{s_{0}(x)}^{L} d s P(x, s) .
\end{aligned}
$$

Since, from the dynamic boundary condition, $P=-\rho U \phi_{s}$, and $\phi \equiv 0$ outside the hull surface,

$$
\begin{aligned}
F_{Y} & =-\rho U \int_{-a(L)}^{b(L)} d x \phi(x, L) \\
& =\rho U \int_{-a(L)}^{b(L)} d x x \phi_{x}(x, L),
\end{aligned}
$$

after an integration by parts. Substituting the expression for $\phi_{x}$ given in equation (3.3),

$$
\begin{aligned}
& F_{Y}= \rho U \int_{-a(L)}^{b(L)} d x x \frac{-1}{\pi(x+a(L))^{\frac{1}{2}}(b(L)-x)^{\frac{1}{2}}} \int_{-a(L)}^{b(L)} \frac{d \xi}{x-\xi} \phi_{y}(\xi, L) \\
& \times(\xi+a(L))^{\frac{1}{2}}(b(L)-\xi)^{\frac{1}{2}} \\
&=-\rho U \int_{-a(L)}^{b(L)} d \xi \phi_{y}(\xi, L)(\xi+a(L))^{\frac{1}{2}}(b(L)-\xi)^{\frac{1}{2}} \\
& \times \frac{1}{\pi} \int_{-a(L)}^{b(L)} \frac{d x}{x-\xi} \frac{x}{(x+a(L))^{\frac{1}{2}}(b(L)-x)^{\frac{1}{2}}} \\
&=-\rho U^{2} \int_{-a(L)}^{b(L)} d \xi \eta_{s}(\xi, L)(\xi+a(L))^{\frac{1}{2}}(b(L)-\xi)^{\frac{1}{2}},
\end{aligned}
$$


since $\phi_{y}=\mathrm{U} \eta_{s}$ and

$$
\frac{1}{\pi} \int_{-a(L)}^{b(L)} \frac{d x}{x-\xi} \frac{x}{(x+a(L))^{\frac{1}{2}}(b(L)-x)^{\frac{1}{2}}}=1 .
$$

As expected, the total lift depends on only the beam and the slope in the direction of motion of the hull at the stern.

Similarly, the starboard-up roll moment, $M_{R}$, about the $s$-axis is given by

$$
\begin{aligned}
M_{R} & =\int_{0}^{L} d s \int_{-a(s)}^{b(s)} d x x P(x, s) \\
& =\frac{\rho U}{2} \int_{-a(L)}^{b(L)} d x x^{2} \phi_{x}(x, L) .
\end{aligned}
$$

Making the substitution for $\phi_{x}$ in this equation gives

$$
\begin{aligned}
M_{R}=-\frac{\rho U}{2} \int_{-a(L)}^{b(L)} d \xi \phi_{y}(\xi, L)(\xi+a(L))^{\frac{1}{2}}(b(L)-\xi)^{\frac{1}{2}} \\
\quad \times \frac{1}{\pi} \int_{-a(L)}^{b(L)} \frac{d x}{x-\xi} \frac{x^{2}}{(x+a(L))^{\frac{1}{2}}(b(L)-x)^{\frac{1}{2}}} \\
=-\frac{\rho U^{2}}{2} \int_{-a(L)}^{b(L)} d \xi \eta_{s}(\xi, L)(\xi+a(L))^{\frac{1}{2}}\left(b(L-\xi)^{\frac{1}{2}}\left\{\xi+\frac{b(L)-a(L)}{2}\right\},\right.
\end{aligned}
$$

since

$$
\frac{1}{\pi} \int_{-a(L)}^{b(L)} \frac{d x}{x-\xi} \frac{x^{2}}{(x+a(L))^{\frac{1}{2}}(b(L)-x)^{\frac{1}{2}}}=\xi+\frac{b(L)-a(L)}{2} .
$$

When the hull slope $\eta_{s}(x, L)$ is symmetric about the midpoint of the stern,

$$
x=\frac{b(L)-a(L)}{2}
$$

the roll moment may be rewritten as

$$
\begin{aligned}
M_{R} & =-\rho U^{2} \frac{b(L)-a(L)}{2} \int_{-a(L)}^{b(L)} d \xi \eta_{s}(\xi, L)(\xi+a(L))^{\frac{1}{2}}(b(L)-\xi)^{\frac{1}{2}} \\
& =\frac{b(L)-a(L)}{2} F_{\mathbf{Y}} .
\end{aligned}
$$

That is, the centre of pressure is laterally located at the point corresponding to the midpoint of the trailing edge and not on the centreplane of the hull. This means that a hull, initially yawed to port, will tend to roll so that the starboard edge rises.

In order to find the longitudinal location of the centre of pressure, it is necessary to evaluate the nose-up pitching moment, $M_{P}$, about the $x$-axis : 


$$
\begin{aligned}
M_{P} & =\int_{0}^{L} d s \int_{-a(s)}^{b(s)} d x s P(x, s) \\
& =\rho U L \int_{-a(L)}^{b(L)} d x \times \phi_{x}(x, L)-\rho U \int_{0}^{L} d s \int_{-a(s)}^{b(s)} d x x \phi(x, s) .
\end{aligned}
$$

Substituting for $\phi_{x}$ gives

$$
\begin{aligned}
M_{P}= & -\rho U^{2} L \int_{-a(L)}^{b(L)} d \xi \eta_{s}(\xi, L)(\xi+a(L))^{\frac{1}{2}}(b(L)-\xi)^{\frac{1}{2}} \\
& +\rho U^{2} \int_{0}^{L} d s \int_{-a(s)}^{b(s)} d \xi \eta_{s}(\xi, s)(\xi+a(s))^{\frac{1}{2}}(b(s)-\xi)^{\frac{1}{2}} .
\end{aligned}
$$

That is,

$$
M_{P}=L F_{Y}+\rho U^{2} \int_{0}^{L} d s \int_{-a(s)}^{b(s)} d x \eta_{s}(x, s)(x+a(s))^{\frac{1}{2}}(b(s)-x)^{\frac{1}{2}} .
$$

The second term in this expression may also be written as

$$
-\int_{0}^{L} d s F_{\mathrm{r}}(s)
$$

where $F_{Y}(s)$ is the longitudinal lift distribution, and is therefore, by necessity, negative. Therefore the centre of pressure will be located forward of the trailing edge, at the point

$$
\bar{s}=L-\int_{0}^{L} d s F_{Y}(s) / F_{Y} .
$$

It is an easy task to calculate the lift force and moments using the above expressions, as the integrals involved either can be evaluated analytically or are quite amenable to numerical integration.

For example, if $\eta_{s}(x, s)$ is dependent only on $s$, that is

$$
\eta_{s}(x, s)=-f(s)
$$

then, for any waterplane shape defined by $a(s)$ and $b(\dot{s})$, the lift force is given by

$$
F_{Y}=\frac{\pi}{8} \rho U^{2} f(L)(b(L)+a(L))^{2},
$$

the roll moment by

$$
M_{R}=\frac{\pi}{8} \rho U^{2} f(L) \frac{b(L)-a(L)}{2}(b(L)+a(L))^{2},
$$

and the pitching moment by 


$$
M_{P}=\frac{\pi}{8} \rho U^{2}\left\{L f(L)(b(L)+a(L))^{2}-\int_{0}^{L} d s f(s)(b(s)+a(s))^{2}\right\} .
$$

Therefore the centre of pressure is located at $(\bar{x}, \bar{s})$, where

$$
\bar{x}=\frac{b(L)-a(L)}{2}
$$

and

$$
\bar{s}=M_{P} / F_{Y}=L-\int_{0}^{L} d s f(s)(b(s)+a(s))^{2} / f(L)(b(L)+a(L))^{2}
$$

As expected, the centre of pressure has its location at the offset corresponding to the midpoint of the trailing edge, $(b(L)-a(L)) / 2$, but at a station forward of the trailing edge.

Since $(b(L)-a(L)) / 2$ is a measure of how far the hull is yawed, the above expressions seem to indicate that both the lift, $F_{Y}$, and the pitching moment, $M_{P}$, are independent of the yaw angle. However, this is not the case, because there is an indirect effect of yaw on the sum $b(L)+a(L)$. For a given hull shape, defined by $\eta(x, s)$, there is a unique waterplane shape, determined by $a(s)$ and $b(s)$. As the hull is yawed, its shape with respect to the coordinate axes changes and, therefore, so does the shape of the wetted region. That is, as the functional form of $\eta(x, s)$ is altered, $a(s)$ and $b(s)$ both change so that equations (3.5) and (3.6) are still satisfied. Therefore the sum $b(L)+a(L)$ does depend on the yaw angle and so do the lift, $F_{Y}$, and the pitching moment, $M_{P}$. This is in contrast to the aerodynamic case in which $F_{Y}$ and $M_{P}$ are independent of the yaw because $b(L)+a(L)$ is constant for a given wing. In both cases, however, the roll moment, $M_{R}$, does depend on the yaw angle.

Neither $F_{Y}$ nor $M_{R}$ depends on the values of $a, b$ and $\eta_{s}$ ahead of the trailing edge. That is, two different hulls will have the same lift and rolling moment if their wetted widths across the trailing edge and their longitudinal hull slopes at the trailing edge are equal. The pitching moment, $M_{P}$, because of the integral term in the expression describing it, does depend on the hull shape at all stations.

\section{A fully-yawed planing hull}

If the hull is further yawed until one of the leading edges becomes a trailing edge, as shown in Fig. 4, the problem is considerably altered. A wake is generated by the trailing edge forward of the stern and there is interaction between the hull and the wake. It is assumed that the starboard edge

$$
x=b(s), \quad 0 \leqslant s \leqslant L,
$$

is always a leading edge and that the port edge, now defined by 


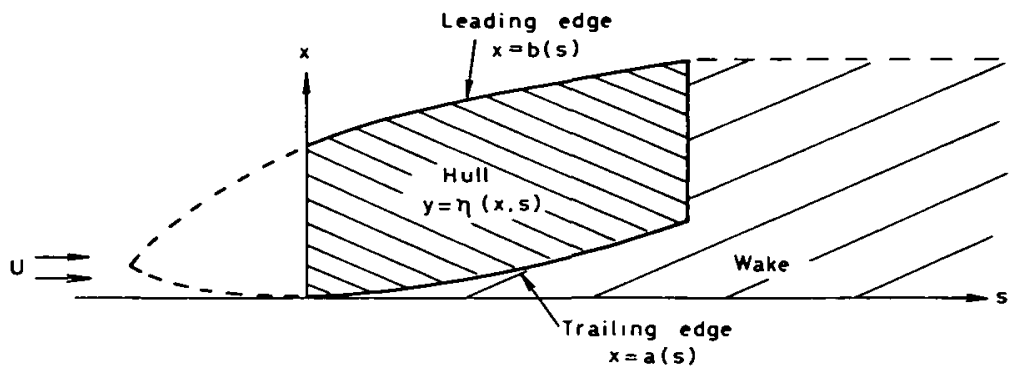

Fig. 4. Waterplane shape for a fully-yawed hull.

$$
x=a(s), \quad 0 \leqslant s \leqslant L,
$$

is always a trailing edge, with $a(0)=0$. As in the last problem, $a(s)$ and $b(s)$ are both non-negative, strictly monotone-increasing functions of $s$, but the added condition $b(s)>a(s), 0<s<L$, is needed here. The wake is assumed to begin at $s=0$ and occupy the area between $x=0$ and the trailing edge, $x=a(s)$. Past the stern, it will be contained in the region between $x=0$ and $x=b(L)$. It is not necessary for the bow to be at $s=0$ (that is, $b(0)$ does not need to be zero), as part of a planing hull which generates no wake may be added for $s<0$ (see Fig. 4).

The mathematical problem is almost identical to that defined in Section 2, requiring solution of the two-dimensional Laplace equation in the lower half-plane, subject to the given conditions. The pressure difference, $P(x, s)$, is zero in the wake region, so the dynamic free-surface condition reduces to

$$
\phi_{s}=0 \text { on } y=0 \text {, }
$$

when $0<x<a(s)$. This implies that

$$
\phi(x, s)=\Phi(x) \text { for } 0<x<a(s)
$$

where $\Phi(x)$ is an unknown function to be determined as part of the solution to the problem. It is also necessary, in this case, for the Kutta condition to be satisfied along the trailing edge, $x=a(s)$, as otherwise a unique solution cannot be obtained. This is "built into" the solution by having a square-root zero in the fluid velocity at $x=a(s)$, thus ensuring that the pressure is continuous across the trailing edge. The problem for $\phi$ is shown in Fig. 5.

The method of solution used in Section 3 is applicable here, the complex function appropriate for this problem being

$$
\Omega(z)=(z-b(s))^{\frac{1}{2}}(z-a(s))^{-\frac{1}{2}} w(z),
$$

where

$$
z=x+i y \quad \text { and } \quad w(z)=\phi_{x}(x, y, s)-i \phi_{y}(x, y, s)
$$


as before, and

$$
\Omega(z)=\Lambda_{x}(x, y, s)-i \Lambda_{y}(x, y, s) .
$$

An inverse square-root singularity in the fluid velocity is still acceptable at the leading edge, $x=b(s)$, but, because of the Kutta condition, this is not the case at the trailing edge. Hence there is the square-root zero in $w(z)$ at $x=a(s)$. The branches of the square-root functions are taken so that

$$
(z-b(s))^{\frac{1}{2}} \rightarrow-i(b(s)-x)^{\frac{1}{2}} \quad \text { as } y \rightarrow 0-, \text { for } x<b(s),
$$

and

$$
(z-a(s))^{-\frac{1}{2}} \rightarrow i(a(s)-x)^{-\frac{1}{2}} \text { as } y \rightarrow 0-, \text { for } x<a(s) .
$$

Thus, in the limit as $y \rightarrow 0-$, equation (5.2) may be written

$$
\Lambda_{x}-i \Lambda_{y}= \begin{cases}(x-b(s))^{\frac{1}{2}}(x-a(s))^{-\frac{1}{2}}\left(\phi_{x}-i \phi_{y}\right) . & x>b(s), \\ -i(b(s)-x)^{\frac{1}{2}}(x-a(s))^{-\frac{1}{2}}\left(\phi_{x}-i \phi_{y}\right), & a(s)<x<b(s), \\ (b(s)-x)^{\frac{1}{2}}(a(s)-x)^{-\frac{1}{2}}\left(\phi_{x}-i \phi_{y}\right), & x<a(s) .\end{cases}
$$

The problem for $\Lambda$ is also shown on Fig. 5 .

Since the conditions of Cauchy's theorem are satisfied,

$$
\Lambda_{y}(x, s)=\frac{1}{\pi} \int_{0}^{b(s)} \frac{d \xi}{x-\xi} \Lambda_{x}(\xi, s)
$$

as shown in Section 3, since there is no contribution to $\Lambda_{y}$ for $\xi<0$ or $\xi>b(s)$ (see Fig. 5). In terms of the original functions,

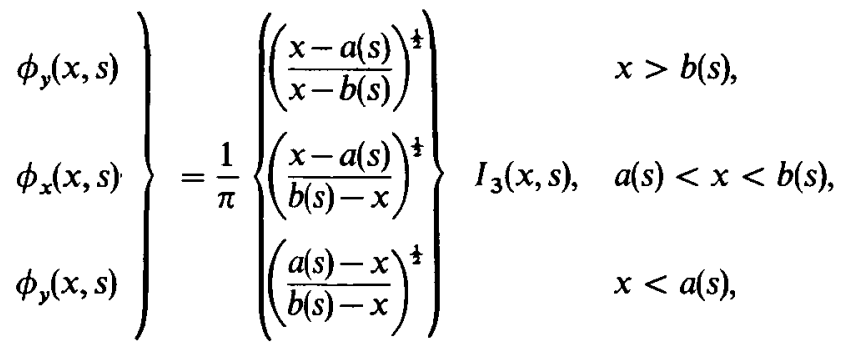

where

$$
I_{3}(x, s)=\int_{0}^{a(s)} \frac{d \xi}{x-\xi}\left(\frac{b(s)-\xi}{a(s)-\xi}\right)^{\frac{1}{2}} \Phi^{\prime}(\xi)-\int_{a(s)}^{b(s)} \frac{d \xi}{x-\xi}\left(\frac{b(s)-\xi}{\xi-a(s)}\right)^{\frac{1}{2}} \phi_{y}(\xi, s) .
$$

If $\Phi(x)$ were known, then the problem would be solved, because expressions for the free-surface elevation for all values of $x$ and $s$ and the loading on the hull (and hence the forces and moments acting on it) could be derived from the above equations for $\phi_{y}$ and $\phi_{x}$. But $\Phi$ is unknown and must somehow be determined before the solution is complete. The following method for finding $\Phi$ was used by Tuck [6] for yawed 


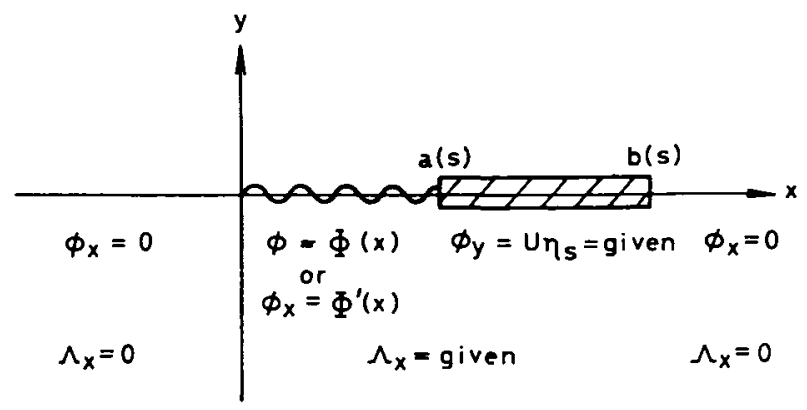

Fig. 5. Cross-flow plane.

slender wings and results in an integral equation which relates $\Phi^{\prime}(x)$, the waterplane shape, and the longitudinal hull slope. The expression for $\phi_{x}$ given in equation (5.3) is integrated from $x=a(s)$ to $x=b(s)$. Since the velocity potential must be continuous at these two points, that is

$$
\begin{gathered}
\phi(a(s), s)=\Phi(a(s)) \quad \text { and } \quad \phi(b(s), s)=0, \\
-\Phi(a(s))=\int_{0}^{a(s)} d \xi\left(\frac{b(s)-\xi}{a(s)-\xi}\right)^{\frac{1}{2}} \Phi^{\prime}(\xi)\left(1-\left(\frac{a(s)-\xi}{b(s)-\xi}\right)^{\frac{1}{2}}\right) \\
-\int_{a(s)}^{b(s)} d \xi\left(\frac{b(s)-\xi}{\xi-a(s)}\right)^{\frac{1}{2}} \phi_{y}(\xi, s),
\end{gathered}
$$

which implies that

$$
\int_{0}^{a(s)} d \xi\left(\frac{b(s)-\xi}{a(s)-\xi}\right)^{\frac{1}{t}} \Phi^{\prime}(\xi)=\int_{a(s)}^{b(s)} d \xi\left(\frac{b(s)-\xi}{\xi-a(s)}\right)^{\frac{1}{t}} \phi_{y}(\xi, s),
$$

or, in terms of the hull slope $\eta_{s}(\mathrm{x}, \mathrm{s})$,

$$
\int_{0}^{a(s)} d \xi\left(\frac{b(s)-\xi}{a(s)-\xi}\right)^{\frac{1}{2}} \Phi^{\prime}(\xi)=U \int_{a(s)}^{b(s)} d \xi\left(\frac{b(s)-\xi}{\xi-a(s)}\right)^{\frac{1}{2}} \eta_{s}(\xi, s) .
$$

This integral equation for determining the unknown wake velocity potential $\Phi(x)$, given the longitudinal hull slope, $\eta_{s}(x, s)$, and the waterplane shape, described by $a(s)$ and $b(s)$, is almost the same as the one obtained by Tuck [6] in his note on yawed slender wings. In that paper, he discusses the mathematical nature of the integral equation and, in certain special cases, presents an analytic solution. The problem is, therefore, to all intents and purposes, solved, as it has been reduced to the task of inverting equation (5.4), and there are numerical techniques available for the solution of such integral equations (see Tuck [6]).

Since $\phi_{y}=U \eta_{s}$, the free-surface elevation caused by the motion of the hull may be written as 


$$
\eta(x, s)= \begin{cases}\frac{1}{\pi} \int_{0}^{s} d \sigma\left(\frac{x-a(\sigma)}{x-b(\sigma)}\right)^{\frac{1}{2}} I_{4}(x, \sigma), & x>b(s), \\ \int_{0}^{s} d \sigma \eta_{\sigma}(x, \sigma)+c_{1}(x), & a(s)<x<b(s), \\ \int_{0}^{s} d \sigma \eta_{\sigma}(x, \sigma)+c_{1}(x)+c_{2}(x, s), & 0<x<a(s), \\ \frac{1}{\pi} \int_{0}^{s} d \sigma\left(\frac{a(\sigma)-x}{b(\sigma)-x}\right)^{\frac{1}{2}} I_{4}(x, \sigma), & x<a(s),\end{cases}
$$

where

$$
\begin{aligned}
& c_{1}(x)=\frac{1}{\pi U} \int_{0}^{s_{0}(x)} d \sigma\left(\frac{x-a(\sigma)}{x-b(\sigma)}\right)^{\frac{1}{2}} \int_{0}^{a(\sigma)} \frac{d \xi}{x-\xi}\left(\frac{b(\sigma)-\xi}{a(\sigma)-\xi}\right)^{\frac{1}{2}} \Phi^{\prime}(\xi) \\
&-\frac{1}{\pi} \int_{0}^{s_{0}(x)} d \sigma\left(\frac{x-a(\sigma)}{x-b(\sigma)}\right)^{\frac{1}{2}} \int_{a(\sigma)}^{b(\sigma)} \frac{d \xi}{x-\xi}\left(\frac{b(\sigma)-\xi}{\xi-a(\sigma)}\right)^{\frac{1}{2}}\left(\eta_{\sigma}(\xi, \sigma)-\eta_{\sigma}(x, \sigma)\right) \\
& \quad-\int_{0}^{s_{0}(x)} d \sigma\left(\frac{x-a(\sigma)}{x-b(\sigma)}\right)^{\frac{1}{2}} \eta_{\sigma}(x, \sigma) \\
& c_{2}(x, s)=\frac{1}{\pi U} \int_{s_{1}(x)}^{s} d \sigma\left(\frac{a(\sigma)-x}{b(\sigma)-x}\right)^{\frac{1}{2}} \int_{0}^{a(\sigma)} \frac{d \xi}{x-\xi}\left(\frac{b(\sigma)-\xi}{a(\sigma)-\xi}\right)^{\frac{1}{2}} \Phi^{\prime}(\xi) \\
&-\frac{1}{\pi} \int_{s_{1}(x)}^{s} d \sigma\left(\frac{a(\sigma)-x}{b(\sigma)-x}\right)^{\frac{1}{2}} \int_{a(\sigma)}^{b(\sigma)} \frac{d \xi}{x-\xi}\left(\frac{b(\sigma)-\xi}{\xi-a(\sigma)}\right)^{\frac{1}{2}}\left(\eta_{\sigma}(\xi, \sigma)-\eta_{\sigma}(x, \sigma)\right) \\
&-\int_{s_{1}(x)}^{s} d \sigma\left(\frac{a(\sigma)-x}{b(\sigma)-x}\right)^{\frac{1}{2}} \eta_{\sigma}(x, \sigma)
\end{aligned}
$$

with $s_{1}(x)$ that value of $s$ for which $x=a(s)$, and

$$
I_{4}(x, s)=\frac{1}{U} \int_{0}^{a(s)} \frac{d \xi}{x-\xi}\left(\frac{b(s)-\xi}{a(s)-\xi}\right)^{\frac{1}{2}} \Phi^{\prime}(\xi)-\int_{a(s)}^{b(s)} \frac{d \xi}{x-\xi}\left(\frac{b(s)-\xi}{\xi-a(s)}\right)^{\frac{1}{2}} \eta_{s}(\xi, s) .
$$

All the above formulae for $\eta(x, s)$ have been derived under the assumption that $\eta(x, 0)=0$. If this is not the case, for example, when a non-wake-generating section is added for $s<0$, then $\eta(x, 0)$ must be added to each of these expressions.

As expected from previous results, the solution to the problem involves an integral equation which relates the planing hull characteristics, namely the waterplane shape, decribed by $x=a(s)$ and $x=b(s)$, and the hull shape represented by $\eta_{s}(x, s)$ and $c_{1}(x)$. But, in this case, the relationship, given in equation (5.6), is more complicated than those derived previously because of the presence of $\Phi(x)$, the velocity potential in the wake, a function which is itself the solution of an integral equation involving the hull characteristics. 
The easiest course of action available is to fix $a(s), b(s)$ and $\eta_{s}(x, s)$ and determine $\Phi^{\prime}(x)$ from equation (5.4). Then $c_{1}(x)$ and $c_{2}(x, s)$ may be found from equations (5.6) and (5.7) respectively. Hence $\eta(x, s)$ may be determined everywhere. However, this is not the situation which arises in practice. Usually $\eta(x, s)$, as given by equation (5.5) for $a(s)<x<b(s)$, is known and it is the shape of the wetted region, that is, $a(s)$ and $b(s)$, which is required. It was shown by Casling [2] that, when the hull has positive curvature in the direction of motion, it is possible for the pressure to fall below atmospheric forward of the stern. This suggests that the flow has separated from the underside of the hull upstream of the stern. In this case, the position, $x=a(s)$, of the trailing edge must be determined as part of the solution to the problem. However, if the hull has zero or negative curvature in the direction of motion, it may be assumed that there is a transom stern along $x=a(s)$. Thus $a(s)$ may be fixed in advance and $\Phi^{\prime}(x)$ and $b(s)$ are the only unknowns. Equations (5.4) and (5.6) are therefore a pair of integral equations to be solved simultaneously for these two unknowns.

For example, suppose a low-aspect-ratio wedge is yawed with respect to the uniform stream. If the hull shape is assumed to have been given initially by

$$
\eta(x, s)=-\alpha s \pm c s, \quad x \gtrless 0,
$$

for small parameters $\alpha$ and $c$, then, once the hull has been yawed through a small angle $\theta$,

$$
\eta(x, s)= \begin{cases}-(\alpha \cos \theta+c \sin \theta) s+(c \cos \theta-\alpha \sin \theta) x, & x>s \tan \theta, \\ -(\alpha \cos \theta-c \sin \theta) s-(c \cos \theta+\alpha \sin \theta) x, & x<s \tan \theta .\end{cases}
$$

There is a change in the longitudinal hull slope, $\eta_{s}(x, s)$, along the line $x=s \tan \theta$ and, since

$$
\alpha \cos \theta-c \sin \theta<\alpha \cos \theta+c \sin \theta
$$

for the values of $\theta$ under consideration, the line $x=s \tan \theta$ acts like a transom stern. That is, the free surface leaves the hull smoothly along the keel line $x=s \tan \theta$. Since $x=a(s)$ describes the position of the trailing edge, $a(s)$ has been fixed and equals $s \tan \theta$. It should be noted that the free surface also leaves the transom stern, now given by

$$
s=-x \tan \theta+L \sec \theta,
$$

smoothly.

When, as in the first case, $a(s)$ is also unknown, three equations are needed in order to obtain a unique solution for $\Phi^{\prime}(x), b(s)$ and $a(s)$. So another condition involving 
one or more of the functions must be found. It comes from the behaviour of the flow at the trailing edge, in the following way. The problem is different from the case of a slightly-yawed hull, because one leading edge has become a trailing edge and the flow must now separate smoothly from the bottom of the hull along this edge $x=a(s)$. Continuity of the free-surface elevation across the trailing edge into the wake region is incorporated into the equations for $\eta(x, s)$, and $\eta_{s}(x, s)$ is continuous along $x=a(s)$ from the Kutta condition. However, for smooth flow detachment to occur, the curvature must remain constant. That is, the curvature of the free surface immediately after separation must equal the curvature of the hull at the separation point (see Oertel [5]). The continuous curvature condition may be derived as follows.

The slope of the free surface is given by

$$
\eta_{s}(x, s)=\left\{\begin{array}{l}
\eta_{s}^{0}(x, s), \quad a(s)<x<b(s) \\
\frac{1}{\pi}\left(\frac{a(s)-x}{b(s)-x}\right)^{\frac{1}{2}}\left\{\int_{0}^{a(s)} \frac{d \xi}{x-\xi} \frac{\Phi^{\prime}(\xi)}{U}\left(\frac{b(s)-\xi}{a(s)-\xi}\right)^{\frac{1}{2}}\right. \\
\left.\quad-\int_{a(s)}^{b(s)} \frac{d \xi}{x-\xi} \eta_{s}^{0}(\xi, s)\left(\frac{b(s)-\xi}{\xi-a(s)}\right)^{\frac{1}{2}}\right\}, x<a(s),
\end{array}\right.
$$

where $\eta_{s}^{0}(\mathrm{x}, \mathrm{s})$ is assumed known. When $x<a(s)$, the above equation may be rewritten as

$$
\begin{aligned}
\eta_{s}(x, s)= & \eta_{s}^{0}(x, s)+\left(\frac{a(s)-x}{b(s)-x}\right)^{\frac{1}{2}}\left\{-\eta_{s}^{0}(x, s)\right. \\
& +\frac{1}{\pi} \int_{0}^{a(s)} \frac{d \xi}{x-\xi} \frac{\left(\Phi^{\prime}(\xi)-\Phi^{\prime}(x)\right)}{U}\left(\frac{b(s)-\xi}{a(s)-\xi}\right)^{\frac{1}{2}} \\
& +\frac{\Phi^{\prime}(x)}{\pi U}\left\{\ln \left|\frac{2(a(s) b(s))^{\frac{1}{2}}+b(s)+a(s)}{b(s)-a(s)}\right|-\left(\frac{b(s)-x}{a(s)-x}\right)^{\frac{1}{2}}\right. \\
& \left.\times \ln \left|\frac{x(a(s)-b(s))}{2(a(s) b(s))^{\frac{1}{2}}(a(s)-x)^{\frac{1}{2}}(b(s)-x)^{\frac{1}{2}}+2 a(s) b(s)-x(a(s)+b(s))}\right|\right\} \\
& \left.-\frac{1}{\pi} \int_{a(s)}^{b(s)} \frac{d \xi}{x-\xi}\left(\eta_{s}^{0}(\xi, s)-\eta_{s}^{0}(x, s)\right)\left(\frac{b(s)-\xi}{\xi-a(s)}\right)^{\frac{1}{2}}\right\} .
\end{aligned}
$$


As $x \rightarrow a(s)$,

$$
\begin{aligned}
\eta_{s}(x, s)=\eta_{s}^{0}(x, s) & +\left(\frac{a(s)-x}{b(s)-x}\right)^{\frac{1}{2}}\left\{-\eta_{s}^{0}(a(s), s)\right. \\
& +\frac{1}{\pi} \int_{0}^{a(s)} d \xi \frac{\left(\Phi^{\prime}(\xi)-\Phi^{\prime}(a(s))\right)}{U} \frac{(b(s)-\xi)^{\frac{1}{2}}}{(a(s)-\xi)^{3 / 2}} \\
& +\frac{\Phi^{\prime}(a(s))}{U} \ln \left|\frac{2(a(s) b(s))^{\frac{1}{2}}+b(s)+a(s)}{b(s)-a(s)}\right| \\
& \left.+\frac{1}{\pi} \int_{a(s)}^{b(s)} d \xi\left(\eta_{s}^{0}(\xi, s)-\eta_{s}^{0}(a(s), s)\right) \frac{(b(s)-\xi)^{\frac{1}{2}}}{(a(s)-\xi)^{3 / 2}}\right\} .
\end{aligned}
$$

In general, the curvature will be finite, and hence continuous, at $x=a(s)$ only if the expression in braces in equation (5.8) vanishes as $x \rightarrow a(s)$, that is, only if

$$
\begin{aligned}
\eta_{s}(a(s), s)=\frac{1}{\pi} \int_{0}^{a(s)} & d \xi \frac{\left(\Phi^{\prime}(\xi)-\Phi^{\prime}(a(s))\right)}{U} \frac{(b(s)-\xi)^{\frac{1}{2}}}{(a(s)-\xi)^{3 / 2}} \\
& +\frac{\Phi^{\prime}(a(s))}{\pi U} \ln \left|\frac{2(a(s) b(s))^{\frac{1}{2}}+b(s)+a(s)}{b(s)-a(s)}\right| \\
& +\frac{1}{\pi} \int_{a(s)}^{b(s)} d \xi\left(\eta_{s}(\xi, s)-\eta(a(s), s)\right) \frac{(b(s)-\xi)^{\frac{1}{2}}}{(\xi-a(s))^{3 / 2}},
\end{aligned}
$$

omitting the superscript on $\eta_{s}^{0}(x, s)$.

Equations (5.4), (5.6) and (5.9) are three integral equations for determining the functions $\Phi^{\prime}(x), a(s)$ and $b(s)$ uniquely for a given hull shape. However, it seems unlikely that such solutions could be obtained in practice, even with the aid of numerical techniques. In fact, it also appears that no solution to the indirect problem (that is, the problem in which $a(s), b(s)$ and $\eta_{s}(x, s)$ are fixed) can be found. The reason for this is that, if $a(s)$ and $b(s)$ are fixed, $\Phi^{\prime}(x)$ is determined uniquely by equation (5.4), but these three functions together may not satisfy the curvature condition, equation (5.9), as well.

\section{Conclusion}

The results presented in this paper highlight the indeterminacy of hull shape in problems which involve the planing motion of a high-speed boat. In practical situations, it is the direct problem of finding the extent of the wetted area for a given hull which is important. It would be very useful, therefore, if a technique for solving this case could be found. However, in the absence of this, progress can be made through the solution of the inverse problem, in which the wetted area is fixed and the hull shape which produced it is determined. 


\section{Acknowledgement}

I would like to thank Professor E. O. Tuck of the University of Adelaide for his encouragement and guidance.

\section{References}

[1] G. S. Baker and G. H. Miliar, "Some experiments un connection with the design of fioats for hydroaeroplanes", British A.R.C. R. \& M., No. 70 (1912).

[2] E. M. Casling, "Planing of a low-aspect-ratio flat ship at infinite Froude number", J. Eng. Math. 12 (1978), 43-57.

[3] E. M. Casling, "Slender planing surfaces", Ph.D. Thesis, University of Adelaide, Adelaide, Australia, 1978.

[4] H. G. Hornung and P. Killen, "A stationary oblique breaking wave for laboratory testing of surfboards", J. Fluid Mech. 78 (1976), 459-480.

[5] R. P. Oertel, "The steady motion of a flat ship, with an investigation of flow near the bow and stern", Ph.D. Thesis, University of Adelaide, Adelaide, Australia, 1975.

[6] E. O. Tuck, "A note on yawed slender wings", J. Eng. Math. 13 (1979), 47-62.

[7] H. Wagner, "The phenomena of impact and planing on water", Z.a.M.M. Bd 12, Heft 4 (1932), 193-215; also NACA Translation 1366.

School of Mathematics and Computer Studies

South Australian Institute of Technology

Ingle Farm

South Australia 5098 